\title{
Analysis of the effect of mobile terminal speed on WLAN/3G vertical handovers
}

\author{
Telemaco Melia* ${ }^{* \ddagger}$, Antonio de la Oliva ${ }^{\dagger}$, Ignacio Soto ${ }^{\dagger}$, Carlos J. Bernardos ${ }^{\dagger}$ and Albert Vidal ${ }^{*}$ \\ *NEC Network Laboratories, Heidelberg, Germany \\ Email: melia,vidal@netlab.nec.de \\ †Departamento de Ingenieria Telematica, Universidad Carlos III de Madrid, Spain \\ Email: aoliva,isoto,cjbc@it.uc3m.es \\ ${ }^{\ddagger}$ Institute of Informatics, University of Goettingen, Goettingen, Germany
}

\begin{abstract}
WLAN hot-spots are becoming widely spread. This, combined with the availability of new multi-mode terminals integrating heterogeneous technologies, opens new business opportunities for mobile operators. Scenarios in which $3 \mathrm{G}$ coverage is complemented by WLAN deployments are becoming available. Thus, true all-IP based networks are ready to offer a new variety of services across heterogeneous access. However, to achieve this, some aspects still need to be analyzed. In particular, the effect of the terminal speed on the detection and selection process of the preferred access network is not yet well understood. In fact, efficiency of vertical handovers depends on the appropriate configuration of mobile devices. In this paper we present a simulation study of handover performance between $3 G$ and WLAN access networks showing the impact of mobile users' speed. The mobile devices are based on the IEEE 802.21 cross layer architecture and use WLAN signal level thresholds as handover criteria. A novel algorithm to dynamically adjust terminals' configuration is presented.
\end{abstract}

\section{INTRODUCTION}

IP mobility across WLAN and $3 \mathrm{G}$ has been widely studied in terms of handover performance. IETF standards are becoming mature and 3GPP scenarios describe how these protocols can be deployed in next generation allIP networks. Approaches such as MIPv6[1], HMIPv6[2], FMIPv6[3], NETLMM ${ }^{1}$, provide mobility management mechanisms, aiming at enabling end to end seamless services across heterogeneous technologies, while minimizing interruptions in ongoing sessions in such networks.

Tomorrow's customers will widely exploit multi-mode terminals (i.e. integrating one or more access technologies) to get better services depending on the environment (e.g. indoor, outdoor). Scenarios in which $3 \mathrm{G}$ coverage is complemented by 802.11 or 802.16 access technologies are becoming available. Upcoming standards, such as IEEE 802.21, propose methods to support mobility across heterogeneous technologies. However, while standards specify functional entities (either implemented in the terminal or in the network) and associated protocol operations, they do not specify configurations of terminal or network components, upon which events are generated.

Considering scenarios in which a terminal can freely move across $3 \mathrm{G}$ and WLAN coverage cells, the configuration of the

\footnotetext{
${ }^{1}$ Network-based Localized Mobility Management, IETF WG
}

terminal for network detection (e.g. WLAN signal level detection) and attachment is a critical issue. For instance, a user preferring WLAN connectivity (when available) over $3 \mathrm{G}$ may need a threshold configuration different from a user preferring 3G. IEEE 802.21 provides a method to configure (upon events or timers) specific thresholds for vertical handovers between $3 \mathrm{G}$ and WLAN. However, the values required for a particular scenario are not specified.

In this paper, through an extensive simulation study, and by using a realistic WLAN signal level path loss model ([4] and [5]), the effect of terminal speed on handover performance has been investigated. In the simulation environment, terminals move according to the random way point model at different speeds. Performance of handovers is measured based on Wireless LAN time utilization, packet loss and number of handovers processed. According to the 802.21 standard, the handover algorithm configures the power thresholds, and then handovers are triggered by signals received from lower layers. In this paper we analyze the effect of the mobile terminal speed into the configuration of the optimal thresholds. The results indicate the configuration to be used depending on the value of the primitive "Link_Configure_thresholds $\rightarrow$ Link speed" of the IEEE 802.21 specification [6]. A potential application scenario, could be a IEEE 802.21 based terminal with builtin GPS devices, that could dynamically adjust thresholds' configuration and sampling techniques for WLAN signal level prediction, according with to speed variation.

A number of papers in the literature [7], [8] and [9] have analyzed performance issues of handovers based on Mobile IP between cellular networks. However these works only study the problems related with upper layers (mainly TCP) due to the differences between the two technologies involved. Some previous works (e.g. [10]) study the integration of WLAN hotspots into $3 \mathrm{G}$ networks; these previous works however, are not based on the 802.21 framework in contrast to ours. The first work, to our best knowledge, that treats the specific problems of inter-technology handovers based on IEEE 802.21 (taking as Mobility handler SIP) is [11]. However, [11] does not analyze the effect of terminal speed. The WLAN signal level model used in this paper is based on the model of [4] and [5], where an accurate study on indoor environments is proposed. We argue that indoors WLAN environments complemented by 
full $3 \mathrm{G}$ coverage will be of a great interest in the future.

The remainder of this paper is organized as follows. Section II introduces the IEEE 802.21 model implemented in the simulator and the handover algorithm developed. Assumptions for both WLAN coverage model and $3 \mathrm{G}$ channel emulations are explained in section III. Section IV covers the obtained results, exploring i) the impact of the speed on the thresholds' configuration (section IV-A), ii) the effect of the RTT ${ }^{2}$ between the Mobile Operator Network and the Mobile Node in the suggested solution (section IV-B) and iii) an analysis of the possible sampling techniques for the signal level depending on the speed of the terminal (section IV-C). The paper concludes with section $\mathrm{V}$.

\section{Terminal ARChitecture}

In this section we present the model we have used in this paper. The section has three parts:

- 802.21 Model

- Modification to the Mobile IPv6 stack

- Handover algorithm

\section{A. 802.21 Model}

The IEEE 802.21 specification defines a middle layer called the Media Independent Handover to centralize functionality related to handover. The Media Independent Handover (MIH) functionality has been implemented in the OMNeT++ ${ }^{3}$ simulation tool. It consists of three elements: the MIH Function, the Service Access Points (SAPs) with their corresponding primitives, and the MIH Function Services (Figure 1). The

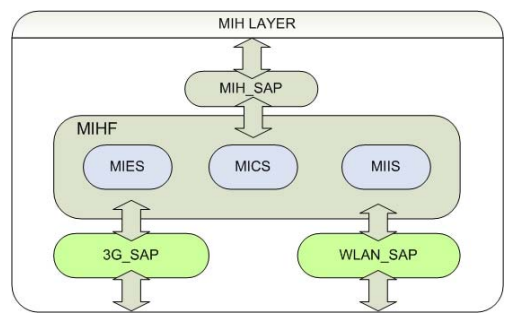

Fig. 1. MIH Architecture

MIH Function (MIHF) is defined in the current IEEE 802.21 specification [6] as a logical entity and the specific MIH implementation of the Mobile Node and the network are not included. In fact, it is important to note that in order to facilitate the overall handover procedure, the MIH Function should be defined following a cross-layer design, allowing the communication with the management plane of every layer within the protocol stack. We have implemented the intelligence of the handover as part of the MIH Function.

The Service Access Points (SAPs) are used to enable the communication between the MIH Function and other layers. In the presented architecture there is one technology independent MIH_SAP which allows the communication between

\footnotetext{
${ }^{2}$ Round Trip Time

${ }^{3}$ http: //www. omnetpp.org
}

the MIH function and upper layers, namely IP, transport, and application. Two technology dependent SAPs are also specified: WLAN_SAP and 3G_SAP, which communicate the MIH Function with the management plane of the 802.11 link layer and the 3GPP link layer, respectively. Note that every SAP defines certain number of primitives that describe the communication with the services in the MIH Function. Since the implemented scenario does not cover all possible use cases, we have only defined here the primitives needed for our scenario.

The MIH Function is supported by three basic services: events (Media Independent Event Service, MIES), commands (Media Independent Command Service, MICS) and information (Media Independent Information Service, MIIS). These services can be defined as local, when the origin and the destination of the service are a single MIH entity; or remote, when the origin and the destination are different MIH entities. Since we focus in this paper on specific scenarios where the terminal does not need to discover neighborhood (Information Services) or to receive remote events/commands from the network, only local communication is taken into account.

The MIH Function has always information up to date of the state of both higher layers and lower layers. Therefore, it will be able to decide when and how a handover procedure has to be performed.

\section{B. Modification to the Mobile IPv6 stack}

In order to have a reasonable control over the handover performance, some modifications to the Mobile IP stack were required.

Mobile IPv6 signaling (Binding Update BU and Binding Acknowledgement BA) sent by a node for WLAN-3G interworking, could be lost in the network before reaching the destination or could be lost in the wireless medium when the Mobile Node has poor signal conditions. Taking into account that the signaling is always sent through the new link in our scenario, a signaling loss may occur due to varying WLAN signal conditions when moving from $3 \mathrm{G}$ to WLAN. When a $\mathrm{BU}$ or BACK is lost the handover at layer 3 is supposed to fail. When the handover fails, the state of the signaling flow can be:

- The BU has not arrived at the Home Agent: the packet flow is reaching the Mobile Node through the old link so no packet loss happens (no handover).

- The BU reaches the Home Agent but the BACK is lost: in this case the packet flow starts arriving to the Mobile Node through the new link.

Binding Updates are usually retransmitted upon timeout. If a BA is not received after a timeout expiration, a retransmission is scheduled and the next timeout is set to the double of the original one. This policy is kept until the timeout reaches a maximum (MAX_BINDACK_TIMEOUT is 32 seconds as specified in the Mobile IP RFC [1]).

Since the Mobile Node has no way of knowing if the Binding Update has reached the Home Agent or not after a handover failure, the handover algorithm must proceed with an action 
to stabilise its state. This action is to perform a handover to the $3 \mathrm{G}$ leg. The major modification introduced into the Mobile IP stack is about the way the retransmission of the Binding Updates is handled. The retransmission algorithm as specified in [1] has been omitted and replaced by MIH intelligence which takes the required actions (namely rolling back to the $3 \mathrm{G}$ channel) in case BU are lost and timers (about 1.5 seconds) expire.

\section{Handover Algorithm}

Our handover algorithm is based on signal thresholds. It relies on the information provided by the Media Dependent layers and the Mobile IP Layer. The handover algorithm reacts upon the reception of three possible signals, which are:

- RSSI (Received Signal Strength Indicator) sample

- Notification about the status of the handover

- Wireless LAN link off message

The handover algorithm is based on two thresholds. The first one, $3 G \rightarrow W L A N$ threshold, defines the minimum wireless LAN signal level that must be received in the Mobile Node to trigger a handover from the $3 \mathrm{G}$ to the wireless LAN. The second one, $W L A N \rightarrow 3 G$ threshold, defines the wireless LAN signal level below which a handover to the $3 \mathrm{G}$ leg is triggered.

A handover to $3 \mathrm{G}$ can be triggered by two events, when the signal level goes below the $W L A N \rightarrow 3 G$ threshold, or when a wireless Link Off message is received.

After the MICS (Media Independent Command Service) triggers a handover to the Mobile IP Layer, the handover algorithm is not allowed to perform another handover until the reception of a handover status message informing of the last handover result. If a handover is not successful, the algorithm performs a handover to the $3 \mathrm{G}$ part to fix the state of the algorithm. There are different causes for the failure of a handover. The BU may not reach the Home Agent or the BU reaches the Home Agent but the Binding Ack is lost.

Before performing a handover some conditions must be satisfied. The interface should be completely configured, with a global routable IPv6 address and default router (DAD procedure completed) associated. Also, all previous handovers should have been completed. If these conditions are not fulfilled the handover is delayed. In the case of handover to WLAN, if the conditions are not met, the handover is skipped until another signal sample arrives. In the case of handover to $3 \mathrm{G}$, the handover is delayed by a timer, waiting for the conditions to be satisfied. The timer has been fixed to $100 \mathrm{~ms}$ (default period of the beaconing in WLAN).

\section{Simulation Setup}

In this section we describe the simulation setup of our experiments. The handover study is conducted by simulating a Mobile Node attached to the $3 \mathrm{G}$ network and performing several handovers between $3 \mathrm{G}$ and wireless LAN, varying terminal speeds and round trip time on the $3 \mathrm{G}$ leg.

The specific scenario analyzed is based on an indoor environment with a wireless LAN cell and full coverage of $3 \mathrm{G}$ technology. We argue that this represents a scenario that will be a typical deployment in the future. Notice that the paper does not cover the WLAN to WLAN handover case. The reader is referred to [13] for an extensive study of WLAN to WLAN handover which complements the work presented here. The work considers wide space with indoor characteristics (such as an airport) in which the user can move at different speeds.

The Mobile Node speed is varied between $2 \mathrm{~m} / \mathrm{s}$ and $10 \mathrm{~m} / \mathrm{s}$. This value represents an upper limit of the speed expected in the big size indoor scenario. Indeed, all pedestrian speeds are below this threshold.

The movement pattern selected is the Random WayPoint Model. With this model each node moves along a zigzag line from one waypoint to the next one, all the waypoints being uniformly distributed over the movement area.

The traffic studied is a downstream video, with a packet size of 160 bytes at application layer and interarrival packet time of 20 $\mathrm{ms}(83 \mathrm{kbps})^{4} .60$ simulation runs were performed for each experiment. This number was chosen as a tradeoff between simulation time and confidence interval size.

\section{A. WLAN Model}

The standard wireless LAN propagation model defined in OMNeT++ is based on free space losses with shadowing and a variable exponential coefficient. The original model implemented in OMNeT++ is suitable for studies that do not analyse in depth the effect of the signal variation. However, the objective of this paper is to have a realistic wireless LAN model, suitable for indoor scenarios based on empirical results. For this purpose, we used the empirical model in [14], which includes variation in the signal due to shadowing and different absorption rates in the materials of the building. The path loss model is the following:

$$
\begin{aligned}
\text { Losses } & =47.3+29.4 * \log (d)+2.4 * Y_{s} \\
& +6.1 * X_{a} * \log (d)+1.3 * Y_{s} * X_{s} \\
X_{a} & =\operatorname{normal}(0,1) \\
Y_{s} & =\operatorname{normal}(-1,1) \\
X_{s} & =\operatorname{normal}(-1.5,1.5)
\end{aligned}
$$

Where $d$ is the distance between the Access Point and the Mobile Node.

The power transmitted by the AP and Mobile Node are defined in the UMA specification [15]. According to this specification, the AP transmission power is $15 \mathrm{dBm}$ while the Mobile Node transmission power is $10 \mathrm{dBm}$. Following these specifications, the AP antenna gain is set to $0 \mathrm{dBi}$ while the Mobile Node antenna gain is set to $-10 \mathrm{dBi}$. The transmission rate of the wireless LAN is fixed to $11 \mathrm{Mbps}$.

The OMNeT++ wireless model defines two thresholds, the Sensitivity threshold and the Active Scanning threshold. The

\footnotetext{
${ }^{4}$ Notice that usual VoIP codecs generate bit rates around $80 \mathrm{kbps}$ and therefore their traffic pattern is very similar to the simulated one
} 
Sensitivity threshold is the minimum level of signal that the receiver can detect. Real products specifications set this level of signal to $-90 \mathrm{dBm}^{5}$. This is the value that we have used in our simulations.

The Active Scanning threshold defines the signal level at which the wireless card starts scanning for other APs in order to perform a WLAN to WLAN handover. When this level of signal is reached the Mobile Node detaches from the current AP. The IEEE $802.11 \mathrm{~b}$ standard does not specify the value for this threshold, its value being design dependant. In the model presented, this value is set to $-80 \mathrm{dBm}$. This value was selected after analyzing via simulations the maximum variability of the wireless LAN signal model. With this threshold, the Mobile Node will always handover to the $3 \mathrm{G}$ leg before reaching the sensitivity threshold.

\section{B. $3 G$ channel Model}

The 3G channel has been modelled as a PPP channel with a connection time of 3.5 seconds, disconnection time of 100 $\mathrm{ms}$, bandwidth of $384 \mathrm{kbps}$ (downlink) and variable delay of 100 to 150 ms per way.

The above PPP channel models the $3 \mathrm{G}$ channel when the PDP context is activated. These disconnection and connection times were obtained from measurements in different locations of an office building with a commercial UMTS data card. The round trip time is tuned to typical values of delay in this kind of channel under the same conditions. The connection time is measured as the time elapsed between bringing up the card and the moment when an IP address is assigned to the Mobile Node (activation of a PDP context).

Although the above model takes into account the connection time, in our simulations we have assumed that the PDP context is always active, so the value of the connection time does not have any impact. Indeed, our simulations are based on the following two assumptions i) full $3 \mathrm{G}$ coverage and ii) $3 \mathrm{G}$ link always on,which we argue that are realistic assumptions in typical scenarios.

\section{Evaluation}

In this section we present the handover performance study considering the following metrics:

- Wireless Utilization Time

- Number of Handovers

- Packet loss

In a first step, an analysis of the three metrics for different configurations of the thresholds is performed. Speed varies between $2 \mathrm{~m} / \mathrm{s}$ and $10 \mathrm{~m} / \mathrm{s}$ (Section IV-A). In a second step the study is extended by introducing the RTT in the 3G channel as additional variable (Section IV-B). The way which WLAN signal level is evaluated impacts the overall handover performance, for this reason, we have considered (and compared) several measurement techniques (Section IV-C).

\section{A. Effect of the speed in the thresholds configuration}

Figures 2 to 4 show how the algorithm behavior changes depending on the speed. Figures 2 and 3 respectively show
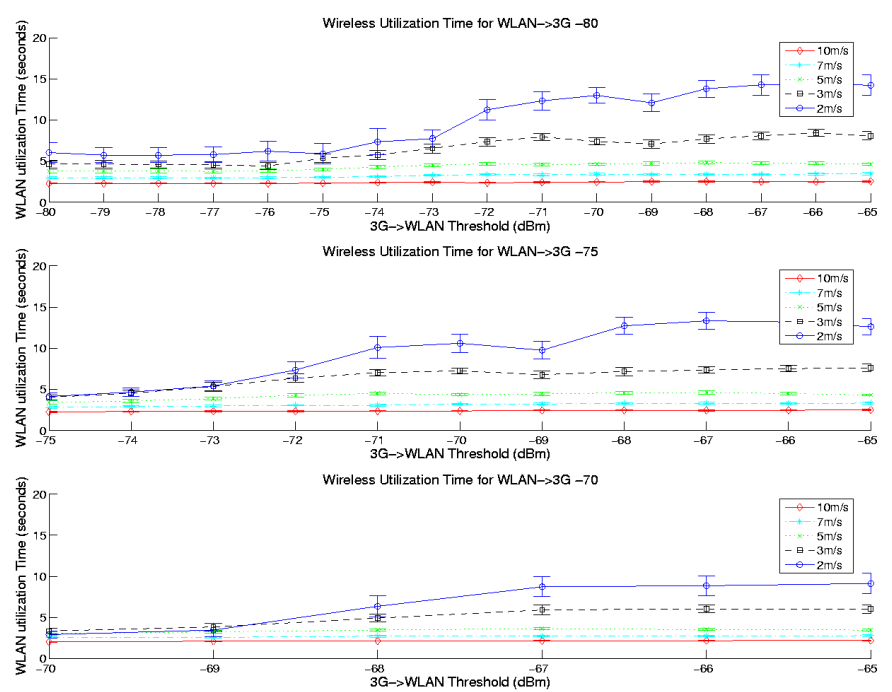

Fig. 2. Wireless Utilization Time for several speeds (RTT 3G 300ms)

the amount of time the Mobile Node is connected to the WLAN and the number of handovers performed by the Mobile Node. It can be observed that while the number of handovers decreases when more stringent thresholds are configured, the Wireless utilization time increases. This shows that the proposed algorithm (based on two thresholds), if properly configured, can optimize the wireless utilization time by reducing the number of useless handovers. Another interesting observation is that as the speed decreases, the difference in the wireless utilization time for different speed values increases.

Figure 4 shows the number of packet losses during the
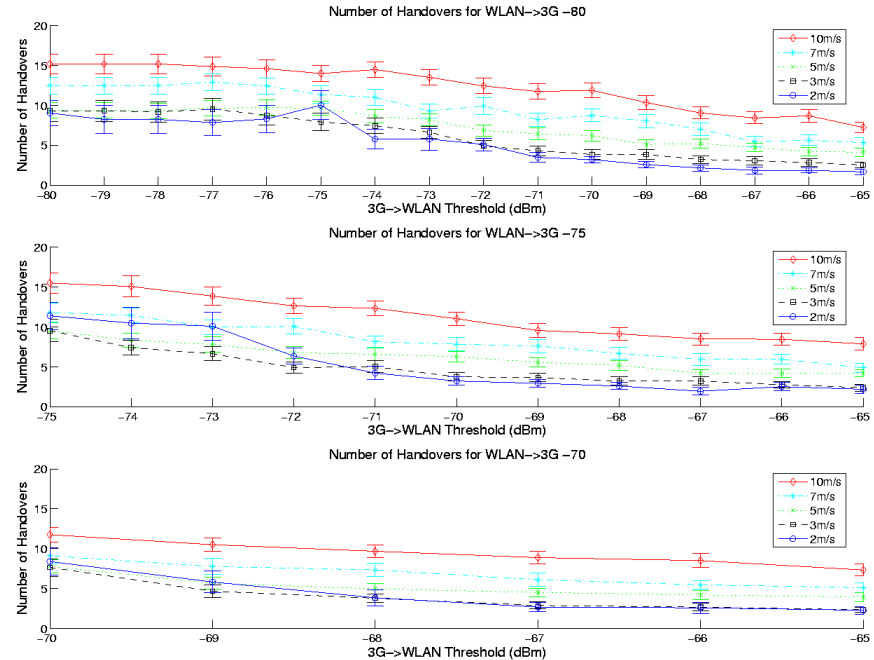

Fig. 3. Number of Handovers for several speeds (RTT 3G 300ms)

handover. All the curves of figure 4 show a common behavior.

${ }^{5}$ SMC Networks SMC2532W-B 
Note that losses can be either due to signal variation or due to handover failure. As the $W L A N \rightarrow 3 G$ threshold increases, losses (both due to signal varation and to a handover failure) are reduced. We define the threshold configuration for zero packet loss, as the configuration of both thresholds in the Mobile Node with which a seamless handover is possible. The threshold configuration for zero packet loss varies for the different speeds. For speed value of $2 \mathrm{~m} / \mathrm{s}$ a configuration of $W L A N \rightarrow 3 G=-70 \mathrm{dBm}$ and $3 G \rightarrow W L A N=-70 \mathrm{dBm}$ is enough to provide zero packet lost. However, for the same threshold configuration and speed about $10 \mathrm{~m} / \mathrm{s}$, on average 20 packets are lost. These values give insightful information for optimal terminal configuration and handover performance.
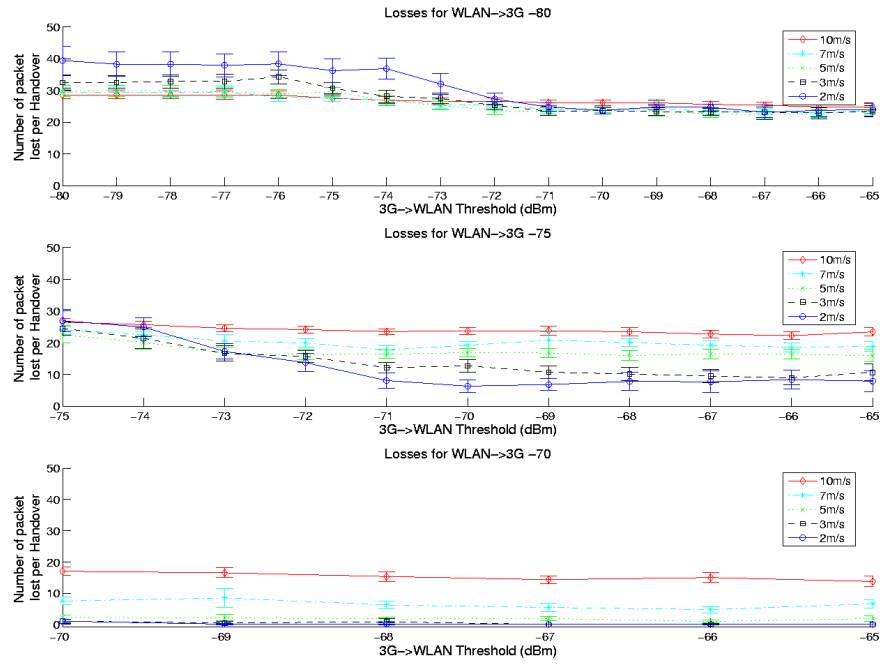

Fig. 4. Number of Packet Lost for several speeds (RTT 3G 300ms)

\section{B. Effect of the $3 G$ channel RTT in the threshold configuration}

To complete the study, an analysis on how the RTT of the 3G link affects the thresholds' configuration is provided. Figure 5 shows how the 3G channel RTT affects the Wireless utilization time, the number of handovers and the packet loss for a specific threshold configuration. Following typical values of RTT for an UMTS channel, which range between $190 \mathrm{~ms}$ and $220 \mathrm{~ms}^{6}$, we vary RTT between $200 \mathrm{~ms}$ and $300 \mathrm{~ms}$ (i.e. the values used in the study are a worse case estimation). Figure 5 shows that RTT affects neither the wireless utilization time nor the number of handovers performed. The major effect is in the number of packets lost. The reason is as follows. Since the RTT increases the time required to handoff to the $3 \mathrm{G}$ leg, the number of packets lost (due to WLAN signal level fading) increases accordingly. The effect is the same as if a less restrictive value for the $W L A N \rightarrow 3 G$ threshold is used.

\section{Effect of the speed in the algorithm for measuring the signal level}

As our handover algorithm is based on signal power thresholds and the signal level can typically vary a lot in indoor

\footnotetext{
${ }^{6}$ Values measured with a commercial data card.
}
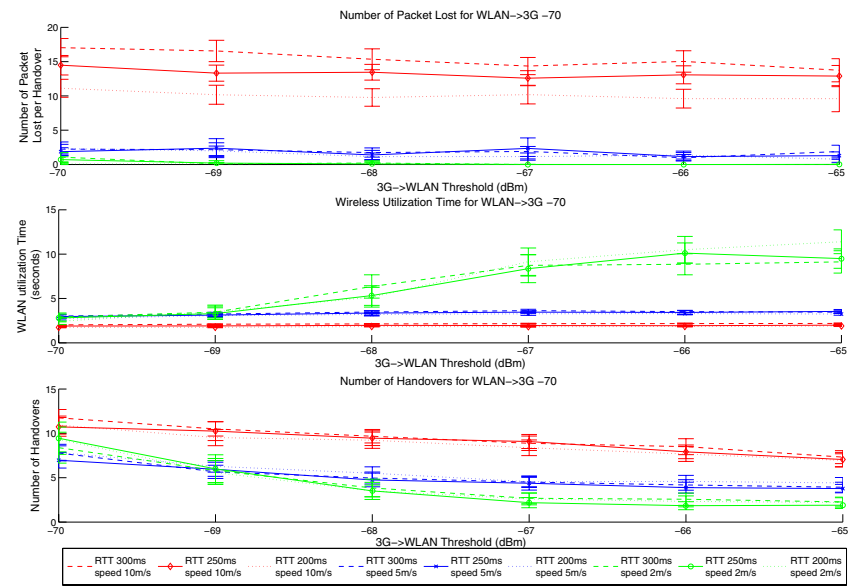

Fig. 5. Wireless Utilization Time, Number of Handovers and Number of Packets Lost for several speeds and RTTs in the 3G Link

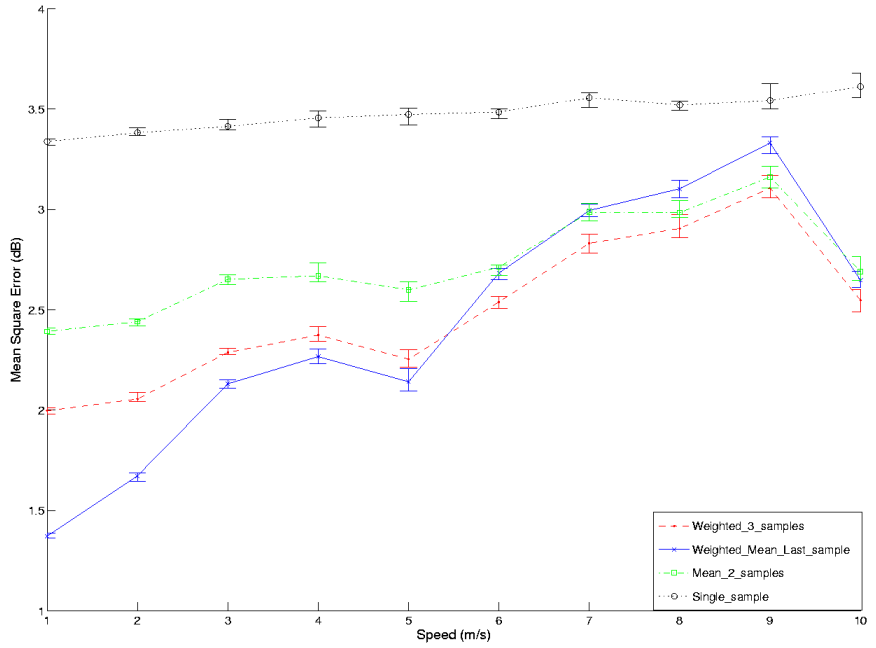

Fig. 6. Mean Square Error of the signal behaviour prediction for different sampling algorithms

environments, the information reaching the MIH layer (e.g. RSSI each beacon interval) can be different in a relative short amount of time. Therefore, taking into account last samples, or a series of samples is not sufficient to derive the trend of the signal conditions. Thus, we propose several approaches to infer the real trend of the signal (based on beaconing interval) against different speed conditions. The different algorithms analyzed are:

- Single Sample (SS): The current value of the signal is the last beacon. $y[n]=x[n]$

- Weighted mean (WM): The current value of the signal is the value given by a weighted mean between the last beacon and the previous one. $y[n]=\alpha x[n-1]+\beta x[n]$

- Weighted mean with the previous mean (WMPM): The current value of the signal is the value given by the weighted mean between the last sample and the last mean. 


\begin{tabular}{|c|c|c|c|c|c|}
\hline \multirow{2}{*}{ Speed } & \multicolumn{2}{|c|}{ WMPM } & \multicolumn{3}{|c|}{ WM3S } \\
& $\alpha$ & $\beta$ & $\alpha$ & $\beta$ & $\gamma$ \\
\hline $1 \mathrm{~m} / \mathrm{s}$ & 0.2 & 0.8 & & & \\
$2-3 \mathrm{~m} / \mathrm{s}$ & 0.3 & 0.7 & & & \\
$4-5 \mathrm{~m} / \mathrm{s}$ & 0.4 & 0.6 & & & \\
$6 \mathrm{~m} / \mathrm{s}$ & & & 0.4 & 0.4 & 0.2 \\
$7-9 \mathrm{~m} / \mathrm{s}$ & & & 0.5 & 0.3 & 0.2 \\
$10 \mathrm{~m} / \mathrm{s}$ & & & 0.6 & 0.3 & 0.1 \\
\hline
\end{tabular}

TABLE I

OPTIMAL PARAMETERS FOR THE CONFIGURATION OF THE WMPM AND WM3S ALGORITHMS

$y[n]=\alpha y[n-1]+\beta x[n]$

- Weighted mean of three samples (WM3S): The current value of the signal is the weighted mean between the last three samples. $y[n]=\alpha x[n-2]+\beta x[n-1]+\gamma x[n]$

Taking into account the four proposed algorithms, a simulation in Matlab has been performed. For each algorithm, the optimal parameters of the weighted mean have been computed (trying all the combinations) taking into account several speeds. Figure 6 shows the Mean Error square obtained while evaluating the signal level for [SS], [WM],[WMPM] and [WM3S] techniques. From the results, it can be seen that for low speeds the algorithm Weighted mean with the previous mean (WMPM) outperforms the others, while for high speed the algorithm Weighted mean of three samples (WM3S) gives the best performance. Based on these results, we recommend a combined approach dependent on terminal speed. Table I presents the optimal configuration for the two recommended algorithms.

As an additional example, we could consider the case of a terminal moving at $2 \mathrm{~m} / \mathrm{s}$. The optimal sampling technique is the WMPM and the optimal threshold configuration values are $-75 \mathrm{dBm}$ for $W L A N \rightarrow 3 G$ and $-70 \mathrm{dBm}$ for $3 G \rightarrow W L A N$. These values (not affected by the RTT) optimize Wireless LAN utilization time while providing acceptable packet loss rate. In a similar way, results can be derived from the graphs for other speeds and RTT.

\section{CONCLUSIONS}

In this paper we have conducted a performance study on vertical handovers between WLAN and $3 \mathrm{G}$ access technologies. For the terminal design we have proposed an architecture following the upcoming IEEE 802.21 cross layer design and the handover algorithm proposed is based on WLAN signal power thresholds. The simulation has been performed for indoor environments and a realistic WLAN propagation model has been implemented in the simulator. The results obtained show the impact of terminal speed and the RTT between the operator (Home Agent) and the Mobile Node, on the handover performance. Based on these results guidelines for thresholds configuration to achieve zero packet loss have been provided. This is in line with current IEEE 802.21 specifications. Indeed, the standard defines protocol operations to configure thresholds for triggers to be generated, while in this paper we have complemented the standard by finding the optimal thresholds for this configuration. In a environment with variable terminal speeds, signal strength measurements need to adapt to this dynamic environment by using sampling techniques to filter out spurious variations. To this aim, we have found that a combined approach, modifying the sampling algorithm depending on the speed, is the more suitable.

\section{REFERENCES}

[1] D. Johnson, C. Perkins, and J. Arkko, "Mobility Support in IPv6," in RFC 3775. IETF, 2004.

[2] H. Soliman, C. Castelluccia, K. E. Malki, and L. Bellier, "Hierarchical Mobile IPv6 Mobility Management (HMIPv6)," in RFC 4140. IETF, 2005.

[3] E. R. Koodli, "Fast Handovers for Mobile IPv6," in RFC 4068. IETF, 2005.

[4] S. Zvanovec, M. Valek, and P. Pechac, "Results of indoor propagation measurement campaign for WLAN systems operating in $2.4 \mathrm{GHz}$ ISM band," in Antennas and Propagation, 2003. (ICAP 2003). Twelfth International Conference on, 2003.

[5] M. Lott and I. Forkel, "A multi-wall-and-floor model for indoor radio propagation," in Vehicular Technology Conference, 2001. VTC 2001 Spring. IEEE VTS 53rd, vol. 1, May 2001, pp. 464-468.

[6] "Draft IEEE Standard for Local and Metropolitan Area Networks: Media Independent Handover Services (Draft 00.05).” IEEE.

[7] R. Chakravorty, P. Vidales, K. Subramanian, I. Pratt, and J. Crowcroft, "Performance Issues with Vertical Handovers-Experiences from GPRS Cellular and WLAN Hot-spots Integration," in Pervasive Computing and Communications, 2004. PerCom 2004. Proceedings of the Second IEEE Annual Conference on. IEEE, 2004.

[8] R. Chakravorty and I. Pratt, "Performance Issues with General Packet Radio Service," in Journal of Communications and Networks (JCN), 2002.

[9] P. Vidales, C. J. Bernardos, G. Mapp, F. Stajano, and J. Crowcroft, "A Practical Approach for 4G Systems: Deployment of Overlay Networks," in First International Conference on Testbeds and Research Infrastructures for the Development of Networks and Communities, 2005. Tridentcom 2005, Trento, ITALY, February 2005, pp. $172-181$.

[10] M. Buddhikot, G. Chandranmenon, S. Han, Y. W. Lee, S. Miller, and L. Salgarelli, "Integration of 802.11 and Third-Generation Wireless Data Networks," in INFOCOM 2003. Twenty-Second Annual Joint Conference of the IEEE Computer and Communications Societies. IEEE, 2003.

[11] A. Dutta, S. Das, D. Famolari, Y. Ohba, K. Taniuchi, T. Kodama, and H. Schulzrinne, "Seamless Handoff across Heterogeneous Networks An 802.21 Centric Approach," in IEEE WPMC, 2005.

[12] E. Wu, J. Lai, and A. Sekercioglu, "An Accurate Simulation Model for Mobile IPv6 Protocol," in Proceedings of Australian Telecommunications, Networks and Applications Conference ATNAC04, December 2004.

[13] C. J. Bernardos, I. Soto, J. Moreno, T. Melia, M. Liebsch, and R. Schmitz, "Experimental evaluation of a handover optimization solution for multimedia applications in a mobile IPv6 network," European Transactions on Telecommunications, vol. 16, no. 4, pp. 317-328, April 2005.

[14] J. Lei, R. Yates, L. Greenstein, and H. Liu, "Wireless Link SNR Mapping Onto An Indoor Testbed," in First International Conference on Testbeds and Research Infrastructures for the Development of Network and Communities (TRIDENTCOM'05). IEEE Computer Society, 2005.

[15] "Universal Mobile Access (UMA) User Perspective (Stage 1) R 1.0.0." Alcatel, AT\&T Wireless Services, BT PLC, Cingular Wireless LLC, Ericsson AB, Kineto Wireless Inc, Motorola, Nokia, Nortel Networks, O2, Rogers Wireless, Siemens AG, Sony Ericsson, T-Mobile USA. 